\title{
Hyperosmolar Therapy for Diabetic Hyperosmolar Ketoacidosis
}

\author{
Micah LA Heldeweg, Joris R Drossaers, Kenrick Berend \\ Department of Internal Medicine, Curaçao Medical Center, Willemstad, Curaçao
}

Received: 19/12/2021

Accepted: 29/12/2021

Published: 21/01/2022

How to cite this article: Heldeweg MLA, Dossaers JR, Berend K. Hyperosmolar therapy for diabetic hyperosmolar ketoacidosis. EJCRIM 2022;9: doi:10.12890/2022_003135.

Conflicts of Interests: The authors declare there are no competing interests.

This article is licensed under a Commons Attribution Non-Commercial 4.0 License

\section{ABSTRACT}

Hyperglycaemic hyperosmolar state (HHS) and diabetic ketoacidosis (DKA) features can occur simultaneously in $27 \%$ of diabetic emergencies and have a two-fold increased risk of death. Despite the high prevalence of this combination, recommended treatments from leading guidelines may not be compatible with the clinical picture.

A 36-year-old man presented with explicit concurrent HHS and DKA. The recommended treatment with simultaneous insulin and volume repletion was followed but resulted in an excessively rapid decline in serum osmolarity. Hyperosmolar therapy ( $\mathrm{NaCl} 3 \%)$ was initiated to mitigate the risk of potentially fatal cerebral osmotic shifts.

The concomitant presence of DKA and HHS leads to a treatment dilemma with a high risk of excessive osmolarity shifts. More evidence is needed, but it is reasonable to initiate tailored treatment to avoid osmolarity reduction rates exceeding the hypernatraemia-based limit of $24 \mathrm{mOsm} / \mathrm{l} /$ day. Hyperosmolar therapy can be considered but requires frequent monitoring of electrolytes and osmolarity.

\section{LEARNING POINTS}

- Simultaneous hyperglycaemic hyperosmolar state (HHS) and diabetic ketoacidosis (DKA) features occur in $27 \%$ of diabetic emergencies and have an almost three-fold increased risk of death.

- Combined HHS and DKA requires simultaneous insulin and volume repletion, which may result in an excessive decline in serum osmolarity. More evidence is needed, but it is reasonable to avoid osmolarity reduction rates above the hypernatraemia-based limit of $24 \mathrm{mOsm} / \mathrm{l} / \mathrm{day}$.

- $\quad$ Consider hyperosmolar therapy $(\mathrm{NaCl} 3 \%)$ to mitigate the risk of potentially fatal cerebral osmotic shifts.

\section{KEYWORDS}

Hyperosmolar hyperglycaemic state, diabetic ketoacidosis, osmolarity, cerebral oedema, case report

\section{INTRODUCTION}

Isolated hyperglycaemic hyperosmolar state (HHS) and diabetic ketoacidosis (DKA) are managed equally in most guidelines. However, this might not be appropriate as pathogenesis and patient characteristics are different. In addition, morbidity and mortality increase substantially when both are present. This case report discusses management and pitfalls in a patient with simultaneous HHS and DKA.

\section{CASE DESCRIPTION}

A 36-year-old man with a medical history of diabetes mellitus type 1 without end-organ damage was brought to the emergency department with intractable vomiting, abdominal pain and progressive lethargy for 3 days. He used subcutaneous injections of $18 \mathrm{U}$ short-acting insulin aspart twice daily. Prior to the development of symptoms, he had intermittent complaints of polyuria and polydipsia without weight loss. He denied drug abuse or excessive alcohol intake. 


\section{of Case Reports in \\ Internal Medicine}

On examination, the patient was well-groomed, dehydrated, lethargic and disoriented, but responsive to pain. He had an acetone foetor and Kussmaul respiration at 34 breaths per minute, and an axillary temperature of $36.5^{\circ} \mathrm{C}$. Vital signs showed a blood pressure of $70 / 40 \mathrm{mmHg}$ and heart rate of 135 beats per minute, pulse oximetry was $99 \%$ on ambient air. There was mild pain upon palpation of the epigastric region, without rebound tenderness. Neurological examination showed reduced consciousness, without nuchal rigidity or pathological reflexes. There were no signs of gingivitis or balanitis. The remainder of the examination revealed no abnormalities.

Blood chemistry revealed severe hyperglycaemia of $81 \mathrm{mmol} / \mathrm{l}$, with an effective osmolarity of $345 \mathrm{mOsm} / \mathrm{l}$. Haemoglobin was $11.2 \mathrm{mmol} / \mathrm{l}$, whereas $\mathrm{HbA} 1 \mathrm{c}$ was 12.6\%. Arterial blood gas analysis showed a pH of 7.22, pCO2 $1.4 \mathrm{kPa}, \mathrm{pO} 218.7 \mathrm{kPa}$, and HCO3 $4.1 \mathrm{mmol} / \mathrm{l}$. The creatinine level was $275.8 \mu \mathrm{mol} / \mathrm{l}$. Ketone bodies and glucose were strongly positive in the urine. Table 1 shows the course of the biochemical values obtained during the first 36 hours.

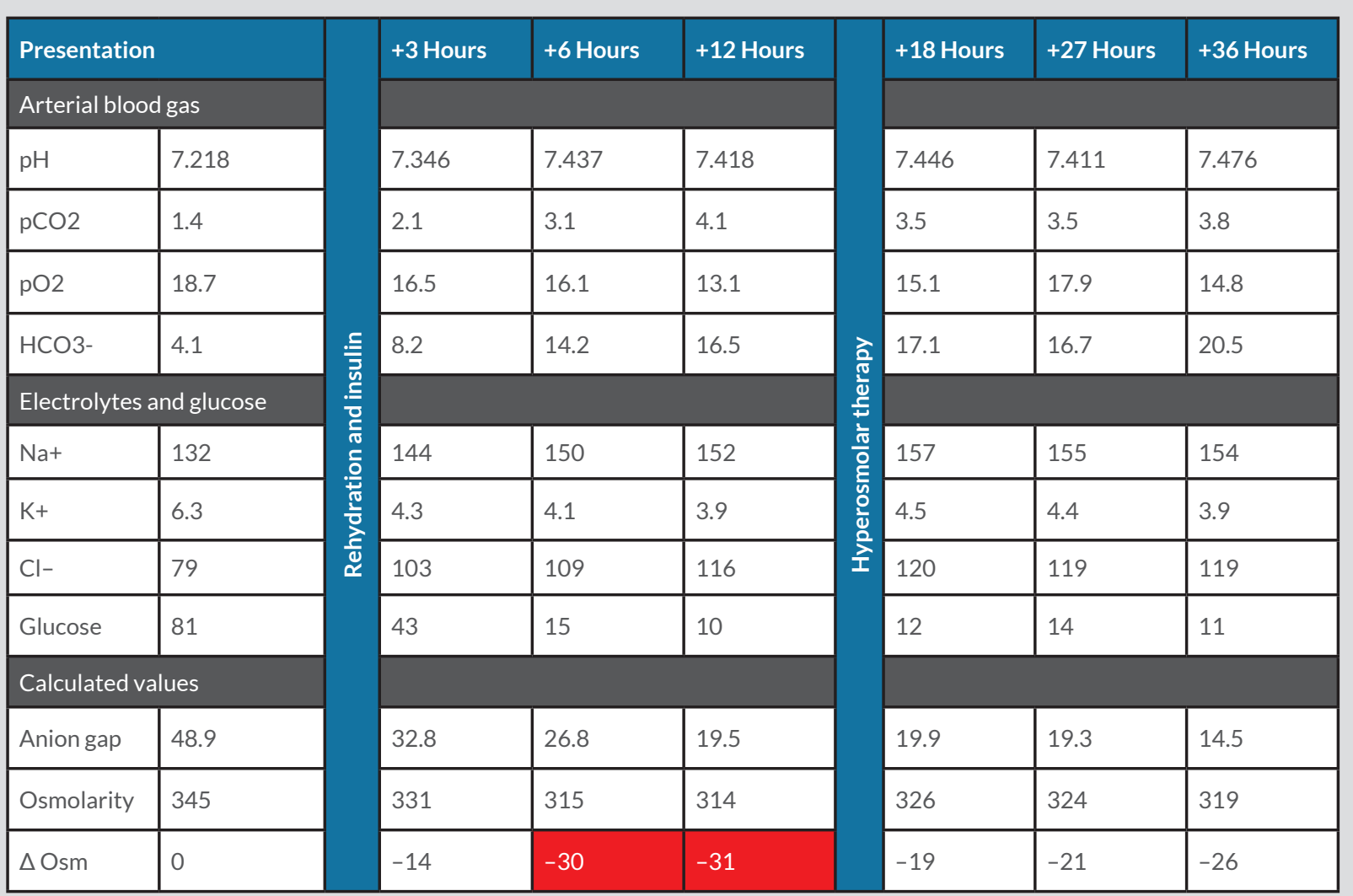

Table 1. Patient's biochemical values during the first 36 hours of admission, after initiation of treatment, and after hyperosmolar therapy.

Gas values in $\mathrm{kPa}$; electrolytes in $\mathrm{mmol} / \mathrm{l} ; \Delta \mathrm{Osm}$, difference in effective osmolarity in $\mathrm{mOsm} / \mathrm{l} ; \Delta$, change in value. Red shading indicates a decrease beyond acceptable limits.

The patient was admitted to the intensive care unit with combined HHS and DKA based on insufficient insulin usage. In adherence to the recommended initial management by the American Diabetes Association (ADA), the patient was treated with an insulin bolus of 0.1 $\mathrm{U} / \mathrm{kg}$ i.v., followed by $0.1 \mathrm{U} / \mathrm{kg} / \mathrm{h}$ and normal saline at $500-1000 \mathrm{ml} / \mathrm{h}$ for the first 2-4 hours. After the initial fluid replenishment, saline infusion was continued at a rate of $4000 \mathrm{ml}$ per 24 hours. This resulted in an osmolarity decrease of $13.5 \mathrm{mOsm} / \mathrm{l}$ in the first 3 hours and 30 $\mathrm{mOsm} / \mathrm{l}$ within 6 hours. This was accompanied by patient drowsiness. We expected a further osmolarity decrease beyond safe limits and so started hyperosmolar therapy after 13 hours with $\mathrm{NaCl} 3 \%$ at a rate of $200 \mathrm{ml} / \mathrm{h}$. As depicted in Fig. 1, the osmolarity increased 12 points to a safer range at 18 hours after presentation and thereafter decreased more gradually. Subsequently a switch to only isotonic fluids and subcutaneous insulin could be made. The patient recovered and was discharged.

\section{DISCUSSION}

A recent retrospective study of 1211 patients found that about $27 \%$ of patients presented with combined features of $\mathrm{HHS}$ and DKA and that this combination resulted in a two-fold increased (odds ratio 2.7 ) mortality risk compared with isolated diabetic emergencies ${ }^{[1]}$. Therefore, it 


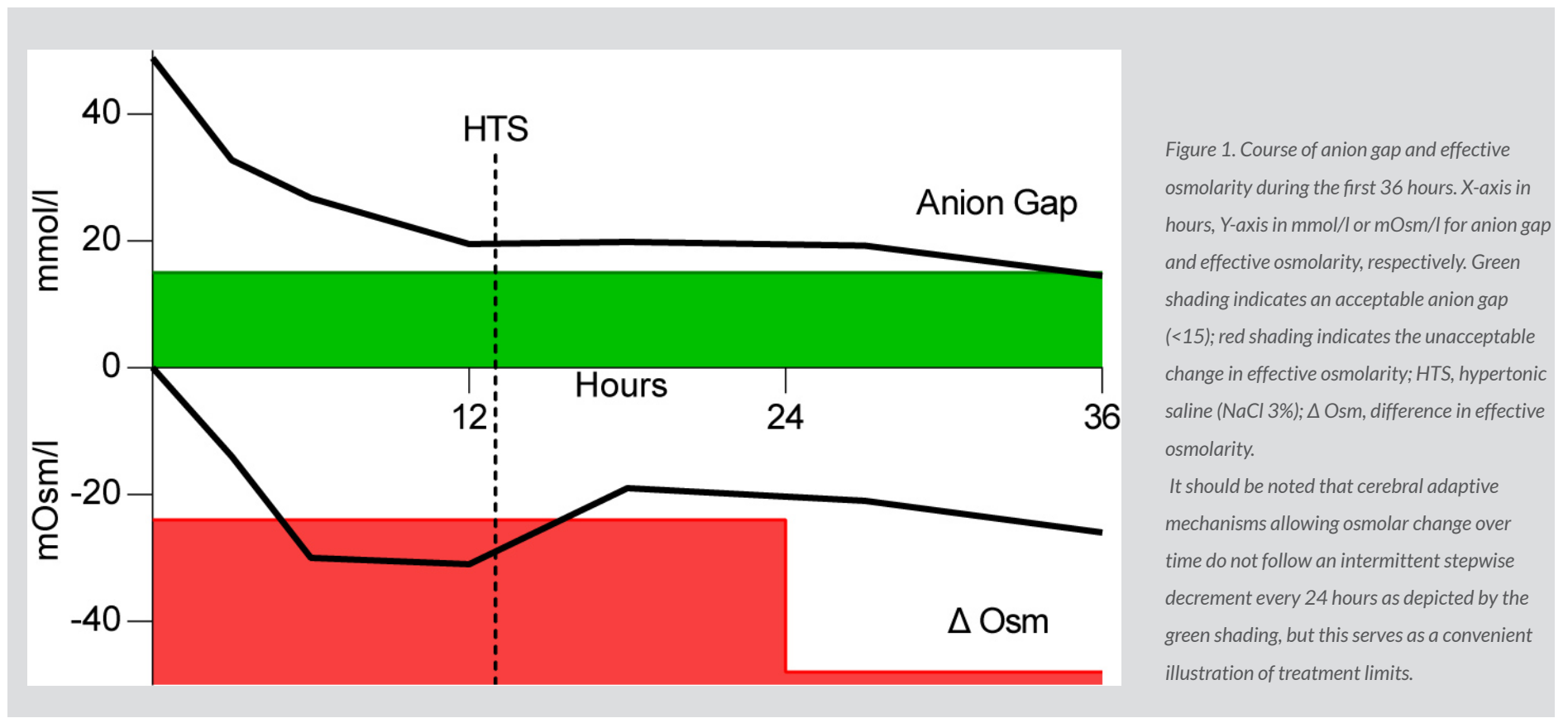

is noteworthy that no prospective studies have determined the best treatment strategies for the management of coexisting HHS and DKA. However, awareness of the pathophysiology of these diabetic complications can guide treatment decisions ${ }^{[2]}$.

DKA develops when absolute insulin deficiency results in loss of lipolysis inhibition. The resulting fatty acid metabolism causes ketone production with acidaemia, the hallmark of DKA. In relative insulin deficiency, there is sufficient insulin to prevent lipolysis, but insufficient for appropriate utilization of glucose, causing its serum concentration to increase gradually. The increase in serum osmolarity draws free water out of the extravascular space that is subsequently lost through diuresis together with glucose and electrolytes causing severe dehydration, the hallmark of HHS. This volume-depleting mechanism occurs in both diabetic emergencies, but to a different degree; the total body water deficit is approximately 6 litres in DKA and 9 litres in HHS. This problem causes renal impairment and a (dilutional) hyponatraemia in both, as water is osmotically shifted from the intracellular to the extracellular space ${ }^{[2]}$.

Both syndromes require volume repletion and insulin, however, the different pathogeneses demand different treatment priorities and considerations. Rapid changes in serum osmolarity during treatment might precipitate cerebral oedema ${ }^{[3]}$. With insulin treatment for DKA, the decrease in serum osmolarity is often gradual; the decrease in serum glucose resolves (dilutional) hyponatremia, protecting the brain from further hazardous osmotic shifts. In HHS, however, extremely high levels of glucose may increase the risk of an overly rapid correction of osmolality if insulin is started at presentation. The combination of HHS and DKA therefore leads to a treatment dilemma because both fluid repletion and insulin therapy may be required initially.

The Joint British Diabetes Societies (JBDS) for Inpatient Care recommends treating HHS without DKA with fluid repletion and delaying insulin therapy until blood glucose levels are no longer falling with fluid therapy alone. The proposed reduction of osmolarity by 3-8 $\mathrm{mOsm} /$ $\mathrm{I} / \mathrm{h}$ equates to an accepted osmolarity reduction of up to $2 \mathrm{mOsm} / \mathrm{l} / \mathrm{h}$. The JBDS states that in concurrent significant ketosis and acidosis, insulin should be started and usage of the guideline should be modified, but no recommendations concerning the limits of osmolality decrease are given ${ }^{[4]}$. The ADA advocates immediate treatment through rehydration and insulin for HHS as well as DKA, and recommends prevention of cerebral oedema through 'avoidance of rapid reduction in serum osmolarity', but interestingly, the preferred rate of reduction is not specified ${ }^{[2]}$.

In patients with hypernatraemia without diabetes, there is a general consensus that sodium correction should not exceed $12 \mathrm{mEq} / \mathrm{l} / \mathrm{day}{ }^{[4]}$. In the effective osmolarity formula, $2 \times$ (sodium+potassium)+glucose, this equates to a maximum decrease in osmolarity of $24 \mathrm{mOsm} / \mathrm{l} / \mathrm{day}$, or $1 \mathrm{mOsm} / \mathrm{l} / \mathrm{h}$.

As shown in Fig. 1, in this case, the rate of osmolarity reduction remained within the JDBS recommendation but exceeded the safe limits for the hypernatraemia-based osmolarity reduction rate. The standard therapy may treat hyperosmolarity and ketoacidosis state but cause a dangerous decrease in osmolarity. We postulated that the administration of hyperosmolar therapy could counterbalance the decrease in effective osmolarity without compromising insulin suppletion. We initiated hyperosmolar sodium infusion ( $\mathrm{NaCl} 3 \%)$ to induce further hypernatraemia and, as demonstrated in Fig. 1, we were able to continue insulin and simultaneously halt the excessively decreasing osmolarity. 


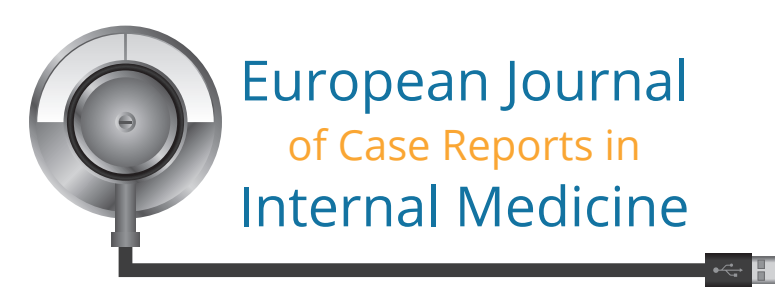

Prospective studies for the management of HHS and DKA are necessary, but in the meantime it seems prudent to avoid large decreases in osmolarity during the first days of treatment. Although saline $3 \%$ may be an option to control the decline in osmolarity, its use is not without risk. No guidelines are established for its use in HHS and DKA and as a $3 \%$ solution has a sodium content of $513 \mathrm{mEq} / \mathrm{l}$, it has to be used with caution in patients with hypertension, heart failure, cirrhosis, renal impairment and oedema. In addition, electrolyte imbalances, a normal anion gap metabolic acidosis, and a prolonged hyperosmolar state may be induced. The use of hypertonic saline should be guided by frequent electrolyte and osmolarity monitoring and the decrease in osmolarity should be probably not exceed $1 \mathrm{mOsm} / \mathrm{l} / \mathrm{hour}$ for the first 48 hours.

\section{REFERENCES}

1. Pasquel FJ, Tsegka K, Wang H, Cardona S, Galindo RJ, Fayfman M, et al. Clinical outcomes in patients with isolated or combined diabetic ketoacidosis and hyperosmolar hyperglycemic state: a retrospective, hospital-based cohort study. Diabet Care 2020;43(2):349-357. https://doi.org/10.2337/dc19-1168.

2. Kitabchi AE, Umpierrez GE, Miles JM, Fisher JN. Hyperglycemic crises in adult patients with diabetes. Diabet Care 2009;32(7):1335-1343. https://doi.org/10.2337/dc099032.

3. Adrogué HJ, Madias NE. Hypernatremia. N Engl J Med 2000;342:1493-1499. https://doi.org/10.1056/NEJM200005183422006.

4. Scott AR, Allan B, Dhatariya K, Flanagan D, Hammersley M, Hillson R, et al. Management of hyperosmolar hyperglycaemic state in adults with diabetes. Diabet Med 2015;32(6):714-724. https://doi.org/10.1111/dme.12757. 